\title{
DCNN based Real-time Adaptive Ship License Plate Recognition (DRASLPR)
}

\author{
Weishan Zhang \\ Department of Software Engineering \\ China University of Petroleum \\ Qingdao, China \\ zhangws@upc.edu.cn \\ Xin Liu \\ School Computer and Communication Engineering \\ China University of Petroleum \\ Qingdao, China \\ 1x@upc.edu.cn \\ Guizhi Min \\ Engineering Technology Research Institute \\ Huabei Oilfield Company, PetroChina \\ Renqiu, China \\ cyy_mgz@petrochina.com.cn
}

\author{
Haoyun Sun \\ Department of Software Engineering \\ China University of Petroleum \\ Qingdao, China \\ hys_upc@163.com
}

\author{
Jiehan Zhou \\ University of Oulu \\ Finland \\ jiehan.zhou@oulu.fi
}

\author{
Zhanmin Zhang \\ Engineering Technology Research Institute \\ Huabei Oilfield Company, PetroChina \\ Renqiu, China \\ cyy_zhangzm@petrochina.com.cn
}

\begin{abstract}
Ship license plate recognition is challenging due to the diversity of plate locations and text types. This paper proposes a DCNN-based (deep convolutional neural network) online adaptive real-time ship license plate recognition approach, namely, DRASLPR, which consists of three steps. First, it uses a Single Shot MultiBox Detector (SSD) to detect a ship. Then, it detects the ship license plate with a designed detector. Third, DRASLPR recognizes the ship license plate. The proposed DRASLPR has been deployed at Dongying Port, China and the
\end{abstract} running results show the effectiveness of DRASLPR.

Index Terms-Ship license plate recognition, Image classification, Online adaptation, Text detection, Deep learning

\section{INTRODUCTION}

Ship license plate recognition [1] [2] is being paid more and more attention in building smart ports for preventing the unlisted ships, stopping the illegal fishing, eliminating potential collisions, and so on. However, it is challenging to accurately recognize ship license plate due to some intrinsic complexities: complex background, weather and light conditions, object occlusion, especially the variety of ship license plate locations and text types.

In literature, there was some existing work using various technologies, e.g., high- resolution range profile based on radar [3], and and high- resolution polarimetric synthetic aperture radar imagery for hierarchical ship detection and recognition [4] [5]. Those radar-based technologies are limited in recognition accuracy and cost.

Surveillance camera provides opportunities for using deep learning, such as DCNN (Deep Convolutional Neural Network) [6] [7] [8] to conduct ship license plate recognition for efficiency and accuracy. Deep learning provides a good choice for background subtraction, license plate localization and recognitio. Masood et al. [9] applied deep learning for plate feature extraction and classification and achieved good performance. The work in [10] applied DCNN for Chinese car license plate recognition and achieved higher accuracy. However, there are few reports on using deep learning for recognizing ship license plate, which has more complex structure than car license plate in China.

Therefore, in this paper, we propose an approach for online adaptive real-time ship plate recognition based on DCNN (DRASLPR), which detects ship license plate with a Deep Convolutional Neural Network, then recognizes the license plate in real time. DRASLPR modifies the SSD (Single Shot MultiBox Detector) for ship license plate localization. We have extensively evaluated the efficiency of DRASLPR. The results show that DRASLPR is able to meet real-time requirement with acceptable accuracy, scalability with high availability.

The remainder of the paper is organized as follows: Section II reviews the related work. Section III presents the DRASLPR architecture and details the system implementation. Section IV evaluates DRASLPR through the on-site deployment. Section $\mathrm{V}$ concludes the paper.

\section{RELATED WORK}

\section{A. Character Recognition}

There are many works on license plate recognition. Gou et al. [11] presented a vehicle license plate recognition method based on character-specific extremal regions (ERs) and hybrid 
discriminative restricted Boltzmann machines (HDRBMs). They used vertical edge detection top-hat transformation, morphological operations, and various validations to detect license plate, extract character-specifics with ERs from detection results, and then recognize the characters by an offline trained pattern classifier of HDRBM.

Hui et al. [2] presented a novel approach to solve the image distortion in ship license plate caused by camera. They used the contour tracking method to remove the redundant information, and then used the Hough transform to detect straight line to speed up the processing speed. A line-fitting algorithm was used for determining the border of a license plate image. Image correction was realized by bilinear space changes. In comparison, DRASLPR uses text line classification for license plate recognition while Hui et al. focused on regulate characteristics angels to improve recognition accuracy.

Lin et al. [12] proposed a method to learn Chinese strokes, radicals and character features based on Convolution Neural Network (CNN) to recognize Chinese Character CAPTCHAs (Completely Automated Public Turing test to tell Computers and Humans Apart), which performed better than most Chinese Optical Character Recognition (OCR) [13]. Lin et al. achieved over 95\% accuracy for single Chinese character and $84 \%$ accuracy for three types of Chinese Character CAPTCHAs with four Chinese characters. However, their method can only work with printed characteristics.

\section{B. Deep learning}

Text detection is a key step for ship license plate recognition, where text detection accuracy significantly affects final recognition accuracy. EAST (An Efficient and Accurate Scene Text Detector) is a simple yet powerful pipeline that yields fast and accurate text detection in natural scenes [14]. It directly predicts words or text-lines of arbitrary orientations and quadrilateral shapes in full images, eliminates unnecessary intermediate steps with fully convolutional network, and achieves an F-score of 0.7820 at $13.2 \mathrm{fps}$ with 720 p resolution. However, EAST focuses on word detection rather than text lines, and at the same time, its multi-orientation detection will suffer high wrong detection rates.

FCN (fully convolutional networks) [15] takes input of arbitrary size and produces correspondingly-sized output with efficient inference and learning. The most extensive application area of FCN is image segmentation. Long et al. [15] demonstrated that fully convolutional networks exceed the state-of-the-art in semantic segmentation. Wang et al. [16] proposed an handwritten string recognition method based on FCN.

\section{DRASLPR APPROACH}

\section{A. Overview}

Figure 1 presents the workflow of the approach.

First, DRASLPR collects and decodes video stream from cameras. Then it locates ship by ship using a SSD (Single Shot MultiBox Detector) [17]. Then, it detects ship license plates from video frames. In ship license plate recognition,

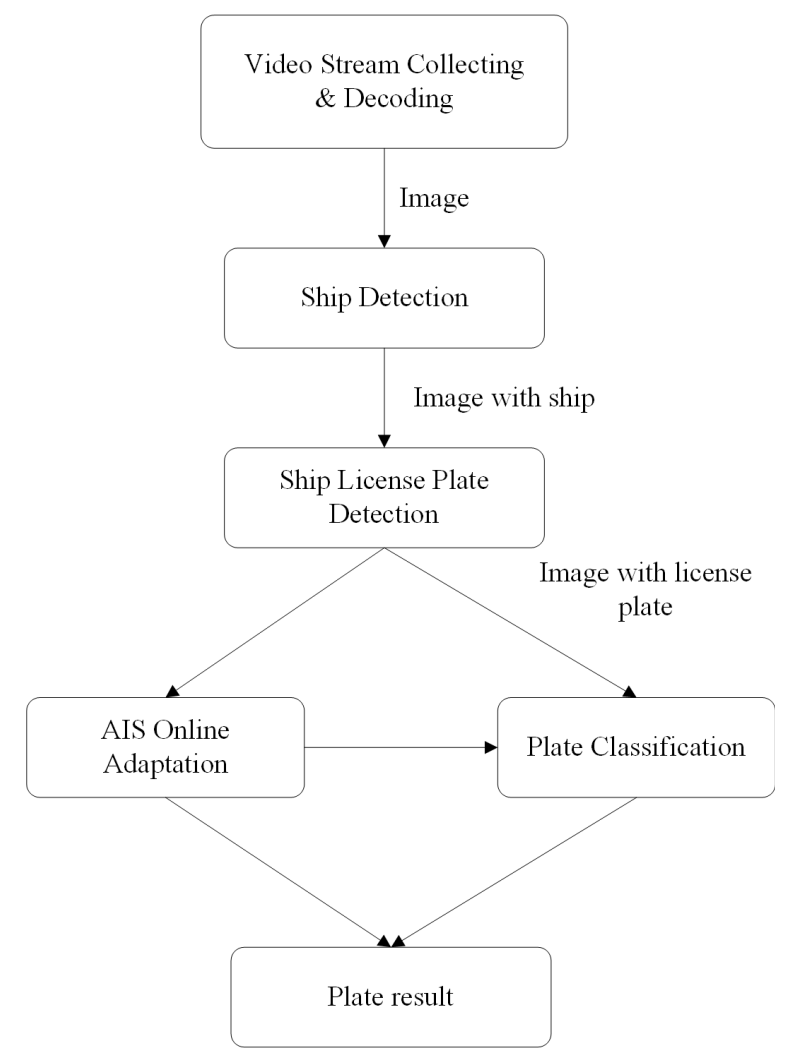

Figure 1: DRASLPR workflow

DRASLPR uses AIS (Automatic Identification System) to get names and locations of ships. When there is only one ship close to port entrance, DRASLPR will record the license plate as the recognition result. When there are more than one ships close to port entrance, DRASLPR first uses a classifier to distinguish the ship license plate, then records the recognition results.

\section{B. Ship detection}

DRASLPR uses SSD (Single Shot MultiBox Detector) to detect ships in a video frame. It uses a small convolutional filter to predict object categories and offsets in bounding box locations, and uses separate predictors (filters) for different aspect ratio detections. These filters are applied to multiple feature maps from the later stages in a network in order to perform detection at multiple scales, as shown in Figure 3. The detection process involves offline training of the SSD network, then the detection process is conducted in an online way.

\section{Ship License Plate Detection}

Considering the following characteristics of ship license plate, we propose a new detection approach based on SSD, which is called SPD (ship plate detector).

- Different from general objects, ship license plate tends to have large aspect ratios, therefore, SPD defines the aspect ratios as long rectangular boxes. 


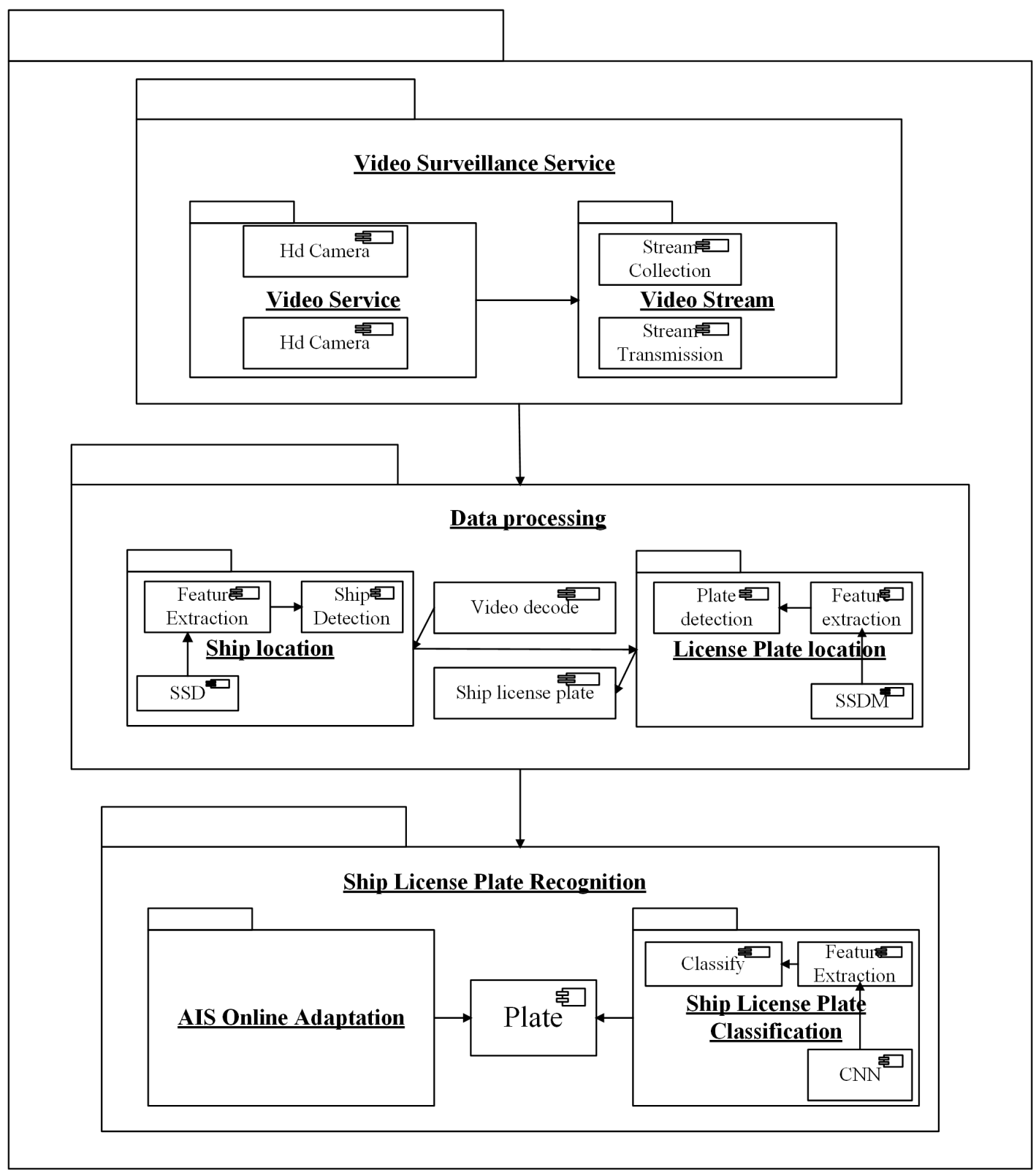

Figure 2: DRASLPR Architecture

- To produce rectangular receptive fields, SPD abandons the standard $3 * 3$ convolutional filters and adopts irregular $1 * 5$ ones, as shown in Figure 4 . These rectangular receptive fields can avoid noisy signals and fit better for ship license plate with larger aspect ratios.

SPD is extensively evaluated as shown in Figure 5(B), and it proves that SPD has better performance than that of SSD (Figure $4(\mathrm{~A})$ ) for ship license plate detection.

\section{Ship License Plate Recognition using a DCNN Classifier}

Due to the limited number of ships in the port and the diversity of ship styles, we propose an online adaptive method combined with AIS information for ship license recognition, which is composed of off-line training and online processing as shown in Figure 6.

When a ship plate is not detected, we collect the ship information (including name and location of the ship) from AIS in an online manner. When a ship is detected, we locate the one closest to the port entrance using AIS, and we record its name as the recognition result. Its accuracy is very high when there only few ships near the port entrance.

We train a ship license plate classifier to distinguish ship license plates. The classifier is a DCNN model, the training procedure for this DCNN classifier is as follows:: 


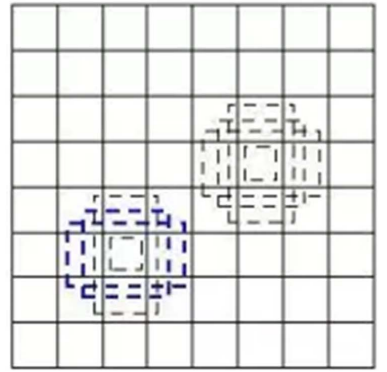

(A) $8 \times 8$ feature map

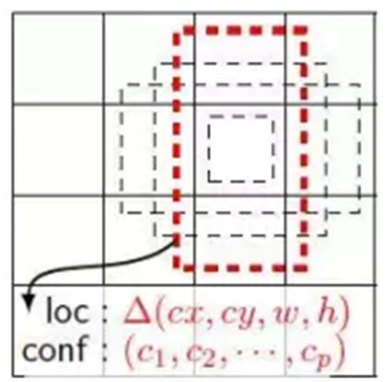

(B) $4 \times 4$ feature map

Figure 3: Multiple feature maps of SSD

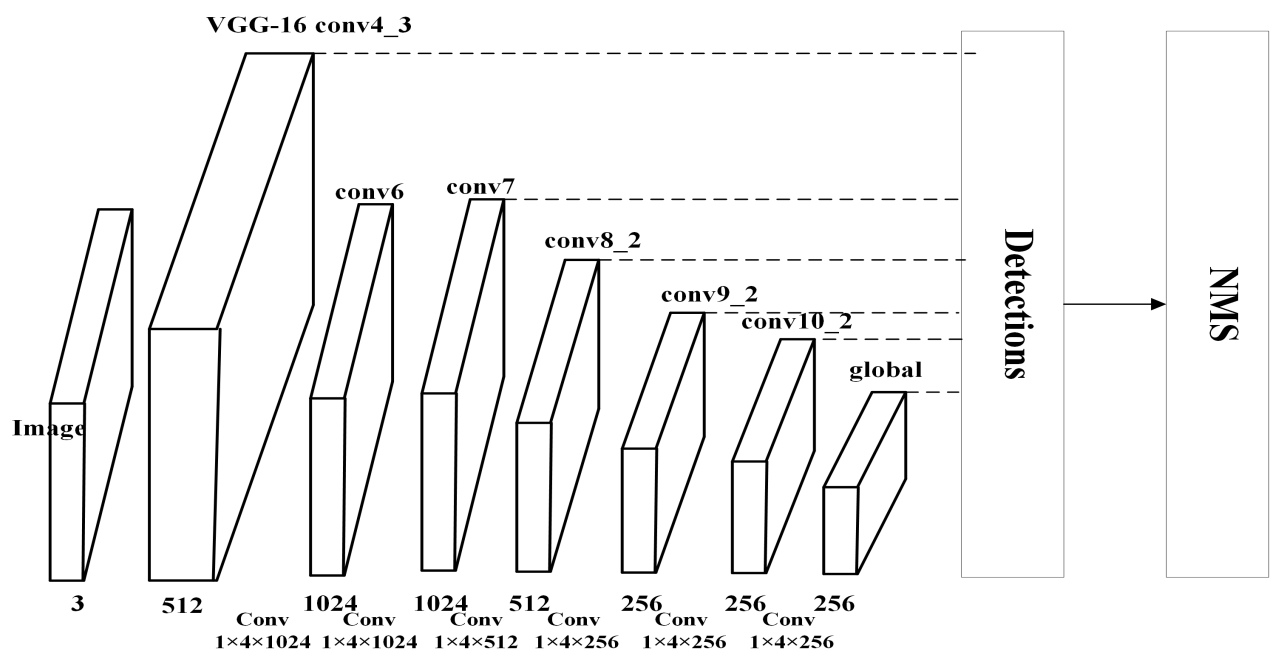

Figure 4: Architecture of SSD based detector

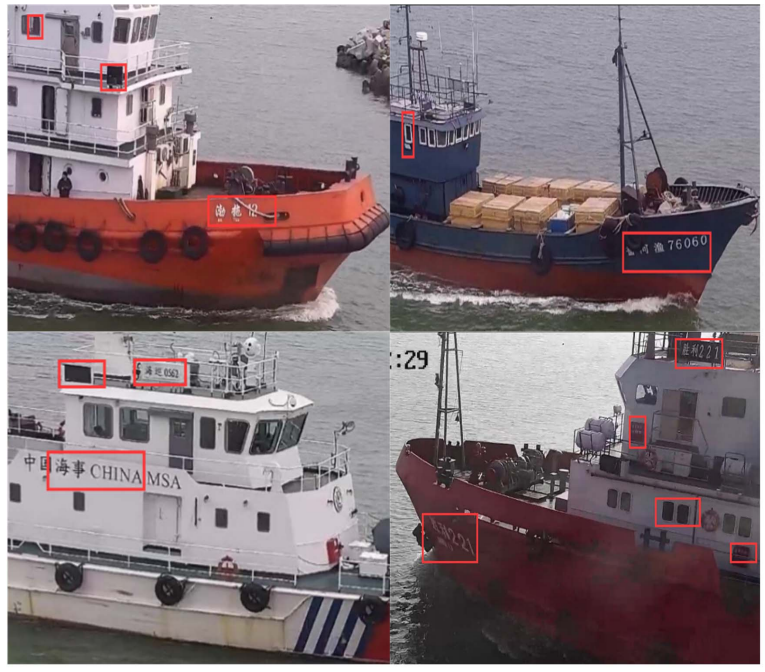

(A) Results of SSD

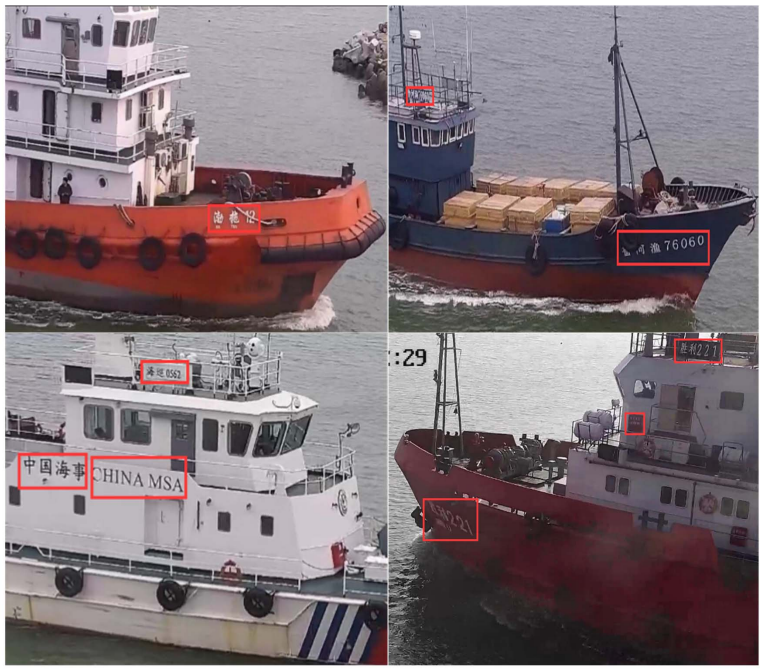

(B) Results of SPD

Figure 5: Test results of SSD and SPD 


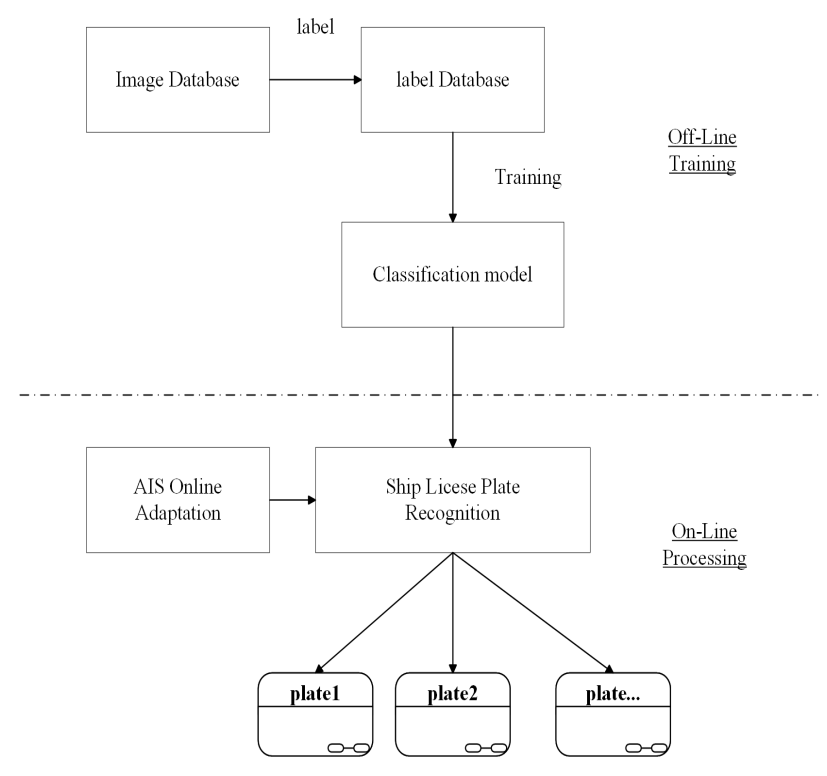

Figure 6: Workflow of ship plate recognition

- Build a ship classification data set. The training data set is the rectangular area of ship plates, and each class represents a ship plate.

- Optimize the classification model. Parameters are adjusted according to intermediate results to obtain an optimal classification model.

When there is no ship or more than one ship close to the entrance in AIS, we use the classifier to recognize the license plates.

\section{E. Implementation Architecture}

The implementation architecture is shown in Figure 2. The key components are SSD-based ship localization, and SPDbased ship license plate detection. The top layer comprises of general video collection and decoding. The second layer is responsible for ship localization, ship license plate detection. The bottom is the Ship License Plate Recognition layer, where an online adaptive method with DCNN based classifier and AIS is used for ship license recognition.

\section{EVALUATION}

We deployed the DRASLPR solution at the port of Dongying, China. The type of video camera used is Hikvision DS2CD3T25D-I5. The pixels used are 1920*1080 and the frame rate is $30 \mathrm{fps}$. The cameras are installed at the both sides of the port entrance with a height of $8 \mathrm{~m}$. The recognition server is a Nvidia $1080 \mathrm{Ti}, 2$ Duo $2.2 \mathrm{GHz}$ desktop computer with 8GB RAM. The running test of the system is presented in Figure 7, which shows the recognized words, direction, time stamps and real time ship image.

\section{A. Performance}

In the experiment, we use the recall- $\mathrm{R}$ and precision-P rates for the evaluation.

$$
R=T P /(T P+F N), P=T P /(T P+F P),
$$

TP refers to true positive, FN refers to false negative, and FP refers to false positive.

We collected 933 video records through one month's running. After re-verifying we found there are 975 ships passing through the port.

For ship localization, we collected 5800 images for training the SSD model in the off-line training phase. The results of field test is shown in Table I. S is the number of ships going through the entrance, S-D means detect records, S-P indicates the positive $S-D, S-T$ refers to the average detection time.

\begin{tabular}{ccccccc}
\hline & S & S-D & S-P & P & R & S-T(ms) \\
\hline Ship Detection & 975 & 963 & 959 & 0.9877 & 0.9836 & 44 \\
\hline
\end{tabular}

Table I: Accuracy of ship localization

Table II shows the performance of ship license plate detection model, which is trained by SPD with 7500 images. $\mathrm{T}$ is the total ships with license plates detected by SSD, T$\mathrm{D}$ means the text records detected from T, D-P indicates the positive T-D, D-T refers to the average text detection time.

\begin{tabular}{ccccccc}
\hline & T & T-D & D-P & P & R & D-T(ms) \\
\hline Plate Detection & 855 & 829 & 784 & 0.9696 & 0.9170 & 39 \\
\hline
\end{tabular}

Table II: Accuracy of license plate detection

Table III lists the result recognition recall and precision. In the test, we collected 6000 images and divided them into 103 categories to train the classifier. T-R means recognized plate, $\mathrm{R}-\mathrm{P}$ indicates the positive $\mathrm{T}-\mathrm{R}, \mathrm{R}-\mathrm{T}$ refers to the average recognition time.

\begin{tabular}{ccccccc}
\hline & D-P & T-R & R-P & P & R & R-T(ms) \\
\hline Text Recognition & 959 & 903 & 889 & 0.9416 & 0.9270 & 31 \\
\hline
\end{tabular}

Table III: Recognition Accuracy

We record the total recall and precision rates for a month for ship license plate recognition as in Table IV.

\begin{tabular}{cccccc}
\hline $\mathrm{S}$ & TP & TR & P & R & T-T(ms) \\
\hline 975 & 903 & 899 & 0.9262 & 0.9221 & 104 \\
\hline
\end{tabular}

Table IV: Accuracy of the proposed approach

It shows that our approach can achieve high accuracy on ship license plate recognition. A complete recognition process only cost average $104 \mathrm{~ms}$, which can fulfill real time requirement. 


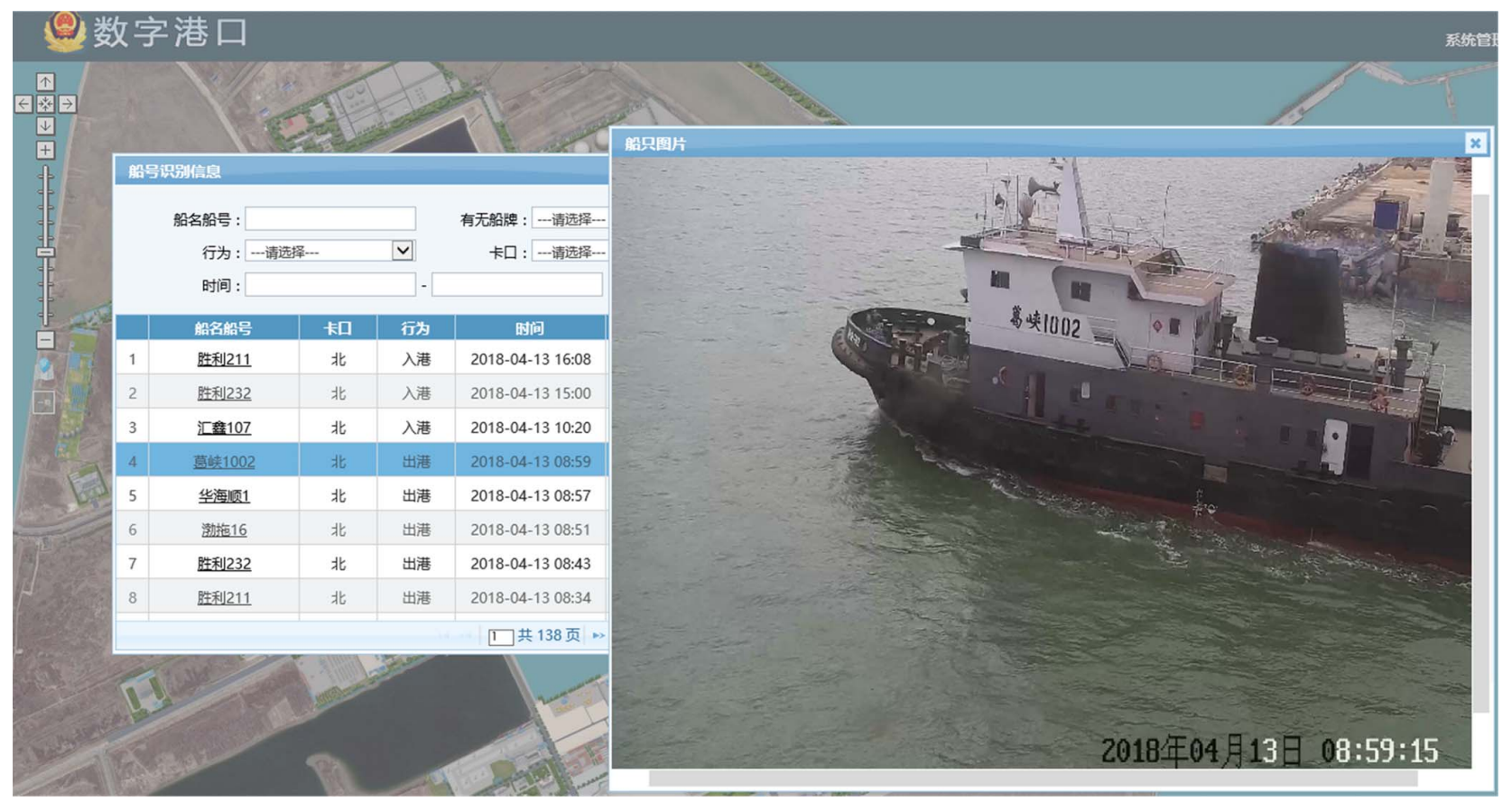

Figure 7: Running of the smart port with intelligent ship plate recognition

\section{CONCLUSION}

In this paper, we propose an online adaptive real-time video processing based approach for ship license plate recognition using different deep learning techniques, and deployed it at the port of Dongying, China. First, it locates the ship using deep convolutional neural network called SSD; then it detects ship license plate by SPD, a specially designed ship license plate detector based on SSD; at last, it recognize the plate with an online adaptive method that combined with a classifier and AIS information. The running results prove that our proposed approach has high recognition recall and precision rates. The system has worked robustly for over a year until now.

\section{ACKNOWLEDGMENT}

This work was supported in part by the Key Research Program of Shandong Province under Grant 2017GGX10140 and in part by the Fundamental Research Funds for the Central Universities(15CX08015A), National Natural Science Foundation of China (No. 61309024).

\section{REFERENCES}

[1] P. M. Alderton, "Port management and operations," Harbors, 2008.

[2] G. U. Hui and Y. Wang, "Study on method of distortion correction in ship license plate recognition system," Modern Electronics Technique, 2009.

[3] J. Lu, Z. Xi, X. Yuan, G. Yu, and M. Zhang, "Ship target recognition using high resolution range profiles based on fmt and svm," in IEEE Cie International Conference on Radar, 2011, pp. 1299-1302.

[4] H. Lang, J. Zhang, and J. Meng, "Hierarchical ship detection and recognition with high-resolution polarimetric synthetic aperture radar imagery," Journal of Applied Remote Sensing, vol. 8, no. 1, p. 083623 , 2014.
[5] A. Maki and K. Fukui, Ship identification in sequential ISAR imagery. Springer-Verlag New York, Inc., 2004.

[6] L. Xu, J. S. J. Ren, C. Liu, and J. Jia, "Deep convolutional neural network for image deconvolution," in International Conference on Neural Information Processing Systems, 2014, pp. 1790-1798.

[7] W. Zhang, P. Duan, W. Gong, Q. Lu, and S. Yang, "A load-aware pluggable cloud framework for real-time video processing," IEEE Trans. Industrial Informatics, vol. 12, no. 6, pp. 2166-2176, 2016. [Online]. Available: https://doi.org/10.1109/TII.2016.2560802

[8] W. Zhang, L. Xu, Z. Li, Q. Lu, and Y. Liu, "A deepintelligence framework for online video processing," IEEE Software, vol. 33, no. 2, pp. 44-51, 2016. [Online]. Available: https: //doi.org/10.1109/MS.2016.31

[9] S. Z. Masood, G. Shu, A. Dehghan, and E. G. Ortiz, "License plate detection and recognition using deeply learned convolutional neural networks," CoRR, vol. abs/1703.07330, 2017.

[10] D. Zang, Z. Chai, J. Zhang, D. Zhang, and J. Cheng, "Vehicle license plate recognition using visual attention model and deep learning," Journal of Electronic Imaging, vol. 24, no. 3, p. 033001, 2015.

[11] C. Gou, K. Wang, Y. Yao, and Z. Li, "Vehicle license plate recognition based on extremal regions and restricted boltzmann machines," IEEE Transactions on Intelligent Transportation Systems, vol. 17, no. 4, pp. 1096-1107, 2016.

[12] D. Lin, F. Lin, Y. Lv, F. Cai, and D. Cao, "Chinese character captcha recognition and performance estimation via deep neural network," Neurocomputing, 2018.

[13] R. Smith, "An overview of the tesseract ocr engine," in International Conference on Document Analysis and Recognition, 2007, pp. 629-633.

[14] X. Zhou, C. Yao, H. Wen, Y. Wang, S. Zhou, W. He, and J. Liang, "East: An efficient and accurate scene text detector," in IEEE Conference on Computer Vision and Pattern Recognition, 2017, pp. 2642-2651.

[15] J. Long, E. Shelhamer, and T. Darrell, "Fully convolutional networks for semantic segmentation," in IEEE Conference on Computer Vision and Pattern Recognition, 2015, pp. 3431-3440.

[16] S. Wang, J. Sun, and S. Naoi, "On study of the reliable fully convolutional networks with tree arranged outputs (TAO-FCN) for handwritten string recognition," CoRR, vol. abs/1707.02975, 2017.

[17] W. Liu, D. Anguelov, D. Erhan, C. Szegedy, S. Reed, C. Y. Fu, and A. C. Berg, "Ssd: Single shot multibox detector," in European Conference on Computer Vision, 2016, pp. 21-37. 